\title{
Authors' reply to Ninan
}

\author{
Edison I O Vidal assistant professor ${ }^{1}$, Fernanda B Fukushima assistant professor ${ }^{2}$, Adriana P Valle \\ associate professor ${ }^{1}$, Ana Teresa A R Cerqueira associate professor ${ }^{3}$, Paulo J F Villas Boas \\ associate professor ${ }^{1}$
}

${ }^{1}$ Internal Medicine Department, Universidade Estadual Paulista (UNESP), 18618-970, Botucatu, SP, Brazil; ${ }^{2}$ Anesthesiology Department, Universidade Estadual Paulista (UNESP), 18618-970, Botucatu, SP, Brazil; ${ }^{3}$ Neurology, Psychiatric and Psychology Department, Universidade Estadual Paulista (UNESP), 18618-970, Botucatu, SP, Brazil

We agree with Ninan that urinary tract infections (UTIs) are often overdiagnosed in older people and that too often asymptomatic bacteriuria is treated with antibiotics. ${ }^{1}$ However, correctly diagnosing UTI in older patients with delirium is more challenging than it may seem, and diagnosing UTI exclusively on the basis of the presence of acute urinary symptoms or bacteriuria accompanied by fever or inflammatory markers would be equally flawed. ${ }^{2}$ For example, in the study cited by Ninan, only $45 \%$ of the patients classified as having a bacteraemic UTI had any urinary tract symptoms. ${ }^{3}$ Moreover, in that study patients with delirium with evidence of a septic illness in the absence of a more likely source (such as pneumonia), and without a negative urine culture, were classified as having presumed UTI. ${ }^{4}$
Competing interests: None declared.

1 Ninan S. Don't assume urinary tract infection is the cause of delirium in older adults. BMJ 2013;346:f3005.

2 Vidal EIO, Villas Boas PJF, Valle AP, Cerqueira ATAR, Fukushima FB. Delirium in older adults. BMJ 2013;346:f2031. (9 April.)

3 Van Duin D. Diagnostic challenges and opportunities in older adults with infectious diseases. Clin Infect Dis 2012;54:973-8.

4 Woodford HJ, George J. Diagnosis and management of urinary tract infection in hospitalized older people. J Am Geriatr Soc 2009;57:107-14.

Cite this as: BMJ 2013;346:f3063

๑ BMJ Publishing Group Ltd 2013 